\title{
Autonomic neuropathy and survival in diabetes mellitus: effects of pancreas transplantation
}

\author{
X. Navarro ${ }^{1}$, W. R. Kennedy ${ }^{1}$ and D. E. R. Sutherland ${ }^{2}$ \\ Departments of ${ }^{1}$ Neurology and ${ }^{2}$ Surgery, University of Minnesota, Minneapolis, Minnesota, USA
}

Summary. Cardiorespiratory reflexes were studied during slow breathing and a Valsalva maneuver in 232 Type 1 (insulin-dependent) diabetic subjects. At 1 to 7 years follow-up, death occurred in $23.4 \%$ of 175 patients with abnormal reflexes and in $3.5 \%$ of 57 patients with normal reflexes. The mortality rates of diabetic subjects with abnormal reflexes were $17 \%$ at 2.5 years, $33 \%$ at 5 years and $40 \%$ at 7 years, significantly higher than for patients with normal reflexes $(4.6 \%, 4.6 \%$ and $13.8 \%)$. Among patients with abnormal reflexes, patients with a functioning pancreas transplantation had better survival rates than patients with a failed transplantation, and in long-term follow-up better than patients without transplantation. A functioning transplantation prevented the progressive decline of cardiorespiratory reflex function that occurred over time in control patients.

Key words: Autonomic neuropathy - Cardiorespiratory reflex - Diabetic neuropathy - Mortality

\section{Introduction}

Cardiorespiratory reflex (CRR) tests can detect abnormal cardiovascular function in as many as $75 \%$ of diabetic subjects (Hilsted and Jensen 1979; Dyberg et al. 1981; Ewing et al. 1985; Low et al. 1986; Zola et al. 1986; Kennedy et al. 1989), often at the time diabetes is first diagnosed (Canal et al. 1978; Pfeifer et al. 1984), even though $27 \%$ or fewer have symptoms of autonomic dysfunction (Kennedy et al. 1989; Canal et al. 1978; Ewing et al. 1980; Young et al. 1983). Moreover, abnormalities on CRR testing are generally considered to be associated with an increased frequency of sudden death (Page and Watkins 1978) and an increased mortality rate (Ewing et al. 1976; Ewing et al. 1980).

Pancreas transplantation reinstitutes a normoglycaemic state in diabetic patients. This suggests that it may have the potential to halt or reverse the effects of diabetes on the nervous system. We therefore investigated the effect of a functioning pancreas transplantation on the CRRs.
We also studied the usefulness of CRR tests as predictors of prognosis and survival in 3 groups of Type 1 (insulindependenddiabetic patients. One group had a functioning pancreas transplantation, a second group had a pancreas transplantation that failed within 3 months and a third group had not received a pancreas transplantation.

\section{Subjects and methods}

\begin{abstract}
We studied all patients with insulin dependent (Type 1) diabetes mellitus whose first CRR test was performed prior to December 31,1986. There were 232 patients, 94 men and 138 women, aged $33.2 \pm 9.2$ years, with a duration of diabetes $20.2 \pm 9.1$ years at entry into the cohort. Pancreas transplantation was performed on 137 patients; all were non-uraemic, either with their native kidneys or having received a kidney transplantation prior to or simultaneous with the pancreas transplantation. CRR tests were performed at the initial visit and at follow-up as described previously (Kennedy et al. 1989). The averaged difference between the highest heart rate (HR) during inhalation and the lowest HR with exhalation during slow breathing at 6 per minute was called the $\Delta R 6$. The highest HR during a Valsalva manoever divided by the lowest rate within $30 \mathrm{~s}$ after the manoever was called the Valsalva ratio (VR). Normal limits were established for each age decade (Kennedy et al. 1989). We also recorded the number of patients who died before December 31, 1987 and the cause of death. Follow-up varied from 12 to 88 months, with 12 patients followed-up longer than 7 years. A pancreas graft was functioning in 53 cases on December 31 , 1987 or at the time of death. The transplant had failed within less than 3 months in 65 patients and at intermediate times in the remaining 19 patients. Ninety-five patients had not received a pancreas transplantation. Survival estimates were calculated using life-table methods and analyzed statistically by the log-rank test. The effects of several variables on survival were analyzed using Cox proportional hazards regression test. Comparisons between groups were made using t-tests and Chi-square tests. Comparisons at several intervals during follow-up were made with the Wilcoxon signed rank test.
\end{abstract}

\section{Results \\ Cardiorespiratory reflexes and mortality}

Both CRR tests were abnormal in 152 patients, one test only was abnormal in 23 and both were normal in 57 . There were statistically significant differences in age $(\mathrm{P}<$ 0.001 ), duration of diabetes $(P<0.001)$, results of $C R R$ 
tests $(P<0.001)$, and degree of sensorimotor neuropathy $(\mathrm{P}<0.05)$ between patients with normal and patients with abnormal results. The duration of follow-up was similar in the two groups.

Of the 232 patients, 43 died (18.5\%) during the period of observation. The cause of death was myocardial infarction for 6 , heart arrest for 4 , heart failure for 2 , pulmonary failure or distress for 4 , liver failure for 4 , kidney failure for 4 , sudden death of unknown origin for 2 , and individual cases with hyperglycaemia, hypoglycaemic coma, pulmonary embolism, lymphoma, anaphylactic shock, viral infection and drug overdose. There was no reliable information on the cause of death for 10 patients.

There were no differences between the deceased and survivors in age or duration of diabetes at entry into the study, but CRR test values were significantly lower for the deceased. In 40 of the deceased both test values were abnormal, one had 1 abnormal value and only 2 had normal CRR values. Mortality rates were $17 \%$ at 2.5 years, $33 \%$ at 5 years and $40 \%$ at 7 years for patients with abnormal CRR tests, and $4.6 \%, 4.6 \%$ and $13.8 \%$ at the same intervals for patients with normal test results. Figure 1 shows the survival curves for patients with normal and with abnormal CRR test results. Survival was significantly lower $(p<0.002)$ for patients with abnormal results. Survival was not associated with sex, age, or duration of diabetes at entry into the study.

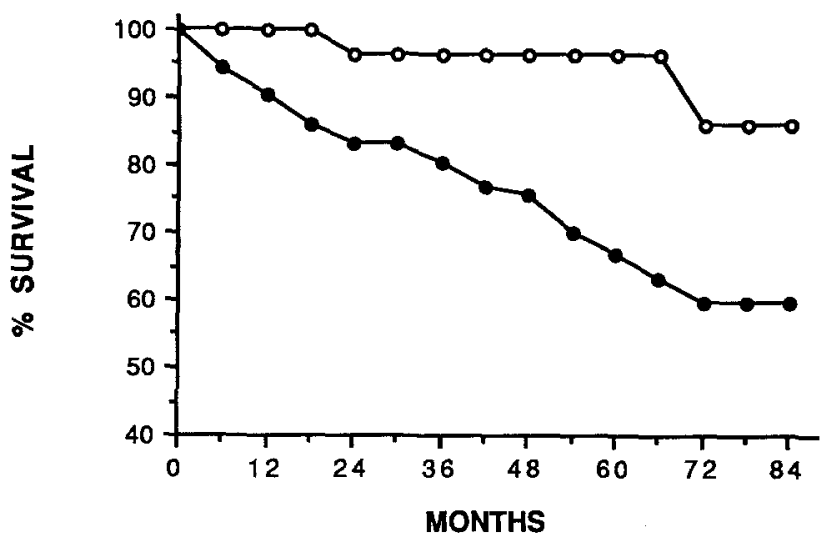

Fig. 1. Survival curves for diabetic patients with (- ) and without $(-\infty)$ ) abnormalities in cardiorespiratory reflex tests (CRR). (Reprinted with permission, Kennedy et al. 1989.)

\section{Influence of pancreas transplantation on survival}

Comparison of patients with a functioning pancreas graft with those whose grafts had failed within 3 months did not show statistically significant differences with respect to age, duration of diabetes or degree of neuropathy (Table 1). Five of 53 patients with a functioning pancreas transplantation had died; the death of 2 was attributed to complications of the surgical procedure or the immunosuppressive treatment. All 5 had abnormal CRR function. Of 65 patients with a failed pancreas transplantation, 22 had died, 9 from causes related to pancreas transplantation. Only one had normal CRR test results. The survival curves of patients with abnormal CRR results, 43 with a functioning pancreas transplantation and 55 with a failed pancreas transplantation, clearly favoured the group with a functioning pancreas transplantation $(p<0.005)$, whether considering all deceased patients (Fig. 2) or excluding those whose death was related to pancreas transplantation ( $40 \%$ in each group). Of the 95 patients without a pancreas transplantation, 62 had abnormal CRR test results. The survival curve of patients with abnormal CRR and a functioning pancreas transplantation was better on long-term follow-up than the curve of these 62 without a pancreas transplantation (Fig.2), despite the risks intrinsic to surgery and immunosuppression and the higher proportion with abnormal CRR results.

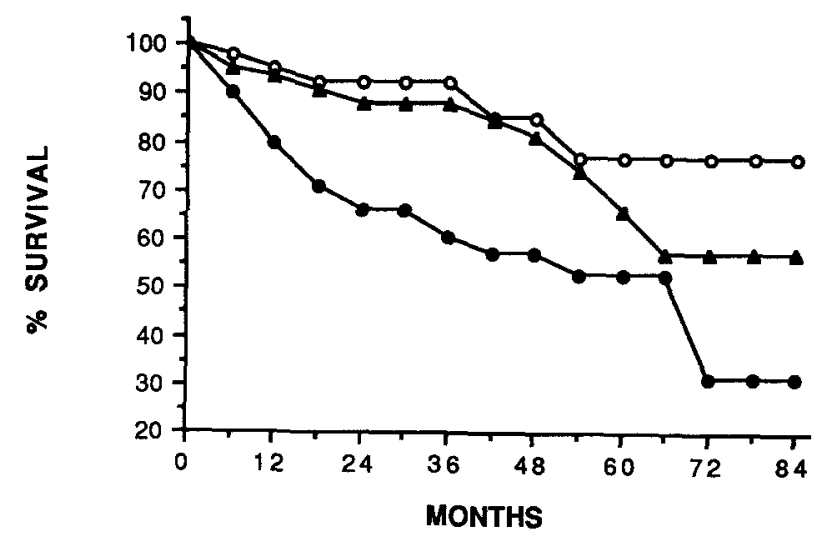

Fig. 2. Survival curves for diabetic patients with abnormalities in cardiorespiratory reflex tests, who had a functioning pancreas transplantation (PTx1 $-\infty-$ ), a failed pancreas transplantation within 3 months (PTx0 - - ), or no pancreas transplantation (NoPTx $\rightarrow-$ ). (Reprinted with permission, Kennedy et al. 1989.)

\section{Influence of pancreas transplantation on cardiorespiratory reflexes over time}

Serially repeated testing during the follow-up time was obtained in 47 patients with a functioning pancreas transplantation and in 36 with either a failed pancreas transplantation or without a pancreas transplantation. The data in Table 2 indicate that autonomic dysfunction worsens significantly with time in the latter group (NoPTx); in contrast, CRR test values remained the same or slightly improved for patients with a functioning pancreas transplantation.

\section{Discussion}

The prevalence of autonomic dysfunction in diabetic patients, judged by abnormalities of cardiovascular reflex responses, is reported from 20 to $75 \%$ (Hilsted and Jensen 1979; Dyberg et al. 1981; Ewing et al. 1985; Low et al. 1986; Zola et al. 1986; Kennedy et al. 1989). The 75\% of our patients with abnormal CRR results is similar to the proportion found in a larger series of 417 patients (Kennedy et al. 1989). This high rate is probably influenced by the characteristics of the population studied. Most of the patients were referred for various therapeutic trials including pancreas transplantation. In the latter, diabetes was usually of long duration and associated with overt secondary complications. 
TABLE 1. Characteristics of 3 groups of diabetic patients according to pancreas transplanation $(\mathrm{PTx})$ status.

\begin{tabular}{llll} 
& & & \\
& PTx \\
& Functioning & $\begin{array}{l}\text { PTx } \\
\text { Failed }\end{array}$ & NoPTx \\
\hline n & 53 & 65 & 95 \\
Sex (f/m) & $36 / 17$ & $41 / 24$ & $46 / 49$ \\
Age (years) & $31.7 \pm 6.2$ & $32.3 \pm 6.3$ & $35.1 \pm 12.1$ \\
Duration of DM (yrs) & $20.5 \pm 6.4$ & $21.1 \pm 6.7$ & $19.4 \pm 12.0$ \\
Follow-up (months) & $38.2 \pm 21.2$ & $38.5 \pm 25.6$ & $39.3 \pm 27.2$ \\
NCS $^{\mathrm{a}}$ abnomal (\%) & 81.1 & 81.6 & 59.7 \\
$\mathrm{CRR}^{\mathrm{b}}$ abnormal (\%) & 81.1 & 84.6 & 65.3 \\
$\Delta 6^{\mathrm{c}}$ & $9.4 \pm 9.9$ & $8.0 \pm 9.6$ & $12.9 \pm 11.1$ \\
$\mathrm{VR}^{\mathrm{d}}$ & $1.38 \pm 0.40$ & $1.28 \pm 0.37$ & $1.40 \pm 0.39$
\end{tabular}

Values are means $\pm \mathrm{SD}$.

a - Nerve Conduction Studies; b - Cardiorespiratory reflexes;

c - Heart rate variation during 6 breaths/min; d - Valsalva ratio

TABLE 2. Cardiorespiratory reflex test results in diabetic patients with (PTx) and without (NoPTx) a functioning pancreas transplant during follow-up time. Comparisons are between the results obtained pre-transplant $(0)$ and at follow-up evaluations of the identical patients of each group. Values are mean \pm s.e.m.

\begin{tabular}{|c|c|c|c|c|c|c|c|c|}
\hline YEARS & 0 & 1 & 0 & 2 & 0 & 3.5 & 0 & 5 \\
\hline PTx & \multicolumn{2}{|c|}{$(47)$} & \multicolumn{2}{|c|}{$(36)$} & \multicolumn{2}{|c|}{ (13) } & \multicolumn{2}{|c|}{$(10)$} \\
\hline$\Delta \mathrm{R} 6^{\mathrm{b}}$ & $8.18 \pm 1.28$ & $8.35 \pm 1.31$ & $8.07 \pm 1.37$ & $9.19 \pm 1.69$ & $10.22 \pm 2.12$ & $10.45^{ \pm} 1.88$ & $10.22 \pm 2.02$ & $8.22 \pm 1.55$ \\
\hline $\mathrm{p}^{\mathrm{a}}$ & \multicolumn{2}{|c|}{0.711} & \multicolumn{2}{|c|}{0.145} & \multicolumn{2}{|c|}{0.552} & \multicolumn{2}{|c|}{0.306} \\
\hline $\mathrm{VR}^{\mathrm{c}}$ & $1.36 \pm 0.08$ & $1.34 \pm 0.06$ & $1.31 \pm 0.06$ & $1.30 \pm 0.06$ & $1.45 \pm 0.12$ & $1.47 \pm 0.11$ & $1.30 \pm 0.06$ & $1.30 \pm 0.05$ \\
\hline $\mathrm{p}^{\mathrm{a}}$ & \multicolumn{2}{|c|}{0.178} & \multicolumn{2}{|c|}{0.303} & \multicolumn{2}{|c|}{0.484} & \multicolumn{2}{|c|}{0.726} \\
\hline NoPTx (n) & \multicolumn{2}{|c|}{ (36) } & \multicolumn{2}{|c|}{ (15) } & \multicolumn{2}{|c|}{ (7) } & \multicolumn{2}{|c|}{ (5) } \\
\hline$\Delta \mathrm{R} 6$ & $6.84 \pm 1.10$ & $5.17 \pm 1.12$ & $9.94 \pm 2.13$ & $8.32 \pm 3.26$ & $7.26 \pm 2.43$ & $4.46 \pm 1.37$ & $8.56 \pm 3.16$ & $4.86 \pm 1.79$ \\
\hline $\mathrm{p}^{\mathrm{a}}$ & \multicolumn{2}{|c|}{0.022} & \multicolumn{2}{|c|}{0.333} & \multicolumn{2}{|c|}{0.273} & \multicolumn{2}{|c|}{0.138} \\
\hline $\mathrm{VR}$ & $1.28 \pm 0.05$ & $1.18 \pm 0.05$ & $1.34 \pm 0.09$ & $1.22 \pm 0.05$ & $1.22 \pm 0.09$ & $1.20 \pm 0.08$ & $1.27 \pm 0.13$ & $1.16 \pm 0.06$ \\
\hline$p^{a}$ & \multicolumn{2}{|c|}{0.001} & \multicolumn{2}{|c|}{0.038} & \multicolumn{2}{|c|}{0.585} & \multicolumn{2}{|c|}{0.104} \\
\hline
\end{tabular}

- calculated $P$ values; ${ }^{b}$ - heart rate variation during 6 breaths $/ \mathrm{min} ;{ }^{c}$ - Valsalva ratio 
The natural history of diabetic autonomic neuropathy is of slow progression. CRR test results are abnormal in a high percentage of patients with symptoms of autonomic dysfunction but also in those who deny symptoms (Kennedy et al. 1989; Canal et al. 1978; Pfeifer et al. 1984; Watkins and Mackay 1980). Once abnormal, CRR function remains so, often deteriorating with time (Ewing et al. 1985; Watkins and Mackay 1980), as also shown in this study. Ewing et al. (Ewing et al. 1976; Ewing et al. 1980) followed patients with symptoms of autonomic neuropathy and with abnormal cardiovascular tests. They found a mortality rate of $44 \%$ at 2.5 years and $56 \%$ at 5 years, much higher than the $6 \%$ and $11 \%$ rates at the same time points in a general diabetic population. Watkins and Mackay (Watkins and Mackay 1980) found that $27 \%$ of 64 diabetic patients with abnormal heart rate variation during slow breathing died during a 5 year follow-up, 4 of sudden death. These results are in general agreement with our findings of mortality rates of $17 \%$ at 2.5 years and $33 \%$ at 5 years. Of our 175 patients with abnormal CRR test results, 41 died during follow-up, whereas only 2 of 57 patients with normal results died. These observations confirm the view that diabetic patients with evidence of autonomic dysfunction have a higher mortality risk than patients with normal test results. A high mortality risk is also reported to be associated with other abnormalities of nerves; such as gastroparesis (Zitomer et al. 1968) and somatic polyneuropathy (Navarro et al. 1990), as well as with other secondary complications of diabetes mellitus, such as vascular disease, nephropathy (Grenfell and Watkins 1986) and retinopathy (Klein et al. 1989).

The relationship between excessive mortality and an abnormal CRR in diabetic patients is not completely understood. An abnormal CRR is often associated with an abnormal blood pressure response to respiratory manoevers and to changes in posture (Ferrer et al. 1990; Kennedy et al. 1991). The frequency of myocardial infarction and the mortality rate (Niakan et al. 1986; Abbot et al. 1988) are known to be high among diabetic patients. Heart disease was the most frequent cause of death $(28 \%)$ in our patients. In addition, approximately one third of diabetic patients show evidence of depressed ventricular function in the absence of ischaemia (Zola et al. 1986). Other abnormalities, found with high prevalence among diabetics with autonomic neuropathy, that should also be considered as possible causes of death are disorders in the reflex control of ventilation (Williams et al. 1984; Sobotka et al. 1986) resulting in sudden death (Page and Watkins 1978), and marked postural hypotension that results in diminished cerebral blood flow in certain circumstances. In our patients renal disease was found to be the cause of death less frequently than in other studies (Ewing et al. 1976; Ewing et al. 1980). This was probably due to the high success rate of kidney transplantation in diabetic patients at our institution for over two decades (Najarian et al. 1989).

Improved glycaemic control by exogenous insulin has not been shown to reverse established symptoms or signs of autonomic neuropathy (Ewing and Clarke 1986; The St. Thomas Diabetic Study Group 1986). No improvement of cardiovascular function tests was found during insulin infusion therapies (Bertelsmann et al. 1987). Treatment with aldose reductase inhibitors was initially thought to improve certain features of autonomic nerve function (Jaspan et al. 1983), but it has not produced significant changes in other studies (Martyn et al. 1987). Although a functioning pancreas graft has not resulted in clear improvement of CRR test results, there are indications that it may slow or halt the progress of the autonomic neuropathy (Solders et al. 1987; Kennedy et al. 1990). In one institution the presence of autonomic neuropathy has been proposed as one inclusion criteria for pancreas transplantation in diabetic patients without end-stage neuropathy (The University of Michigan Pancreas Transplant Evaluation Committee 1988). Our patients with abnormal CRR test results had a better survival rate, if they maintained a functioning pancreas transplantation, than patients who underwent the same surgical procedure unsuccessfully and possibly better than patients without transplantation. At this time therefore, a functioning pancreas graft appears to offer diabetic patients the best chance of arresting the autonomic neuropathy and of prolonging survival.

Acknowledgements. This research was supported by National Institutes of Health grant NS 26348 .

\section{References}

Abbot RD, Donahue RP, Kannel WB, Wilson PWF (1988) The impact of diabetes on survival following myocardial infarction in men vs women. JAMA 260: 3456-3460

Bertelsmann FW, Heimans JJ, Van Rooy JCGM, Dankmeijer HF, Visser SL, Van der Veen EA (1987) Peripheral nerve function in patients with painful diabetic neuropathy treated with continuous subcutaneous insulin infusion. J Neurol Neurosurg Psychiat 50: $1337-1341$

Canal N, Comi G, Saibene V, Musch B, Pozza G (1978) The relationship between peripheral and autonomic neuropathy in insulin dependent diabetes: A clinical and instrumental evaluation. In: Canal N, Pozza G (eds) Peripheral Neuropathies. Elsevier, Amsterdam pp 247-255

Dyberg T, Benn J, Christiansen JS, Hilsted J, Nerup J (1981) Prevalence of diabetic autonomic neuropathy measured by simple bedside tests. Diabetologia 20: 190-194

Ewing DJ, Campbell IW, Clarke BF (1976) Mortality in diabetic autonomic neuropathy. Lancet 1: 601-603

Ewing DJ, Campbell IW, Clarke BF (1980) Assessment of cardiovascular effects in diabetic autonomic neuropathy and prognostic implications. Ann Int Med 92: 308-311

Ewing DJ, Campbell IW, Clarke BF (1980) The natural history of diabetic autonomic neuropathy. Quart J Med 193: 95-108

Ewing DJ, Martyn CN, Young RJ, Clarke BF (1985) The value of cardiovascular autonomic function tests: 10 years experience in diabetes. Diabetes Care 8: 491-498

Ewing DJ, Clarke BF (1986) Diabetic autonomic neuropathy: present insights and future prospects. Diabetes Care 9: 648-665

Ferrer, M.T, Sahinen, F and Kennedy, WR (1990) Baroreflexes in diabetes mellitus. Neurology InPress

Grenfell A, Watkins PJ (1986) Clinical diabetic nephropathy: natural history and complications. Clin Endocrinol Metab 15: 783-805

Hilsted J, Jensen SB (1979) A simple test for autonomic neuropathy in juvenile diabetics. Acta Med Scand 205: 385-387

Jaspan JB, Herold K, Maselli R, Barkus C (1983) Treatment of severely painful diabetic neuropathy with an aldose reductase inhibitor: relief of pain and improved somatic and autonomic nerve function. Lancet 2: $758-762$

Kennedy WR, Navarro X, Sakuta M, Mandell H, Knox C, Sutherland DER (1989) Physiological and clinical correlates of cardiorespiratory reflexes in diabetes mellitus. Diabetes Care 12: 399-408 
Kennedy WR, Navarro X, Goetz FC, Sutherland DER, Najarian IS (1990) Effects of pancreatic transplantation on diabetic neuropathy. N Engl J Med 322: 1031-1037

Kennedy, WR, Navarro, X and Ferrer, T (1991) Cardiovascular responses to tilting in healthy and diabetic subjects. J Neurol Sci In Press

Klein R, Moss SE, Klein BEK, DeMets DL (1989) Relation of ocular and systemic factors to survival in diabetes. Arch Int Med 149: 266272

Low PA, Zimmerman BR, Dyck PJ (1986) Comparison of distal sympathetic with vagal function in diabetic neuropathy. Muscle \& Nerve 4: 592-596

Martyn CN, Reid W, Young RJ, Ewing DJ, Clarke BF (1987) Six-month treatment with sorbinil in asymptomatic diabetic neuropathy: failure to improve abnormal nerve function. Diabetes $36: 987-990$

Najarian JS, Kaufman DB, Fryd DS, McHugh L, Mauer SM, Ramsay RC, Kennedy WR, Navarro X, Goetz FC, Sutherland DER (1989) Long-term survival following kidney transplantation in 100 Type 1 diabetic patients. Transplantation 47: 106-113

Navarro X, Kennedy WR, Loewenson RB, Sutherland DER (1990) Influence of pancreas transplantation on cardiorespiratory reflexes, nerve conduction, and mortality in diabetes mellitus. Diabetes 39: 802-806

Niakan E, Harati Y, Rolak R, Comstock JP, Rokey R (1986) Silent myocardial infarction and diabetic cardiovascular autonomic neuropathy. Arch Int Med 146: 2229-2230

Page MB, Watkins PJ (1978) Cardiorespiratory arrest and diabetic autonomic neuropathy. Lancet 1: 14-16

Pfeifer MA, Weinberg CR, Cook DL, Reenan A, Halter JB, Ensinck JW, Porte D Ir (1984) Autonomic neural dysfunction in recently diagnosed diabetic subjects. Diabetes Care 7: 447-453

St. Thomas Diabetic Study Group, The (1986) Failure of improved glycaemic control to reverse diabetic autonomic neuropathy. Diabetic Med 3: 330-334

Sobotka PA, Liss HP, Vinik AI (1986) Impaired hypoxic ventilatory drive in diabetic patients with autonomic neuropathy. J Clin Endocrinol Metab 62: 658-663

Solders G, Wilczek H, Gunnarsson R, Tyden G, Persson A, Groth CG (1987) Effects of combined pancreatic and renal transplantation on diabetic neuropathy: a two-year follow-up study. Lancet 2: 12321235

University of Michigan Pancreas Transplant Evaluation Committee, The (1988) Pancreatic transplantation treatment for IDDM. Proposed candidate criteria before end-stage diabetic nephropathy. Diabetic Care 11: 669-675

Watkins PJ, Mackay JD (1980) Cardiac denervation in diabetic neuropathy. Ann Int Med 92: 304-307

Williams JF, Morris AI, Hayter RC, Ogilvie CM (1984) Respiratory responses of diabetics to hypoxia, hypercapnia and exercise. Thorax 39: 529-534

Young RJ, Ewing DJ, Clarke BF (1983) Nerve function and metabolic control in teenage diabetics. Diabetes $32: 142-147$

Zitomer BR, Gramm HF, Kozak GP (1968) Gastric Neuropathy in diabetes mellitus: clinical and radiologic observations. Metabolism 17: 199-211

Zola B, Khan JK, Juni JE, Vinik AI (1986) Abnormal cardiac function in diabetic patients with autonomic neuropathy in the absence of ischemic heart disease. J Clin Endocrinol Metab 63: 208-214

Dr. W.R. Kennedy

Box 187 UMHC

University of Minnesota Hospital

420 Delaware Street S.E.

Minneapolis, MN 55455

USA 